\title{
Speech Enhancement by Spectral Subtraction Method
}

\author{
Kaladharan N \\ Assistant professor \\ Department of Electrical Engineering \\ Annamalai University \\ Annamalai Nagar
}

\begin{abstract}
Speech enhancement aims to improve speech quality and intelligibility by using various techniques and algorithms. Speech signal is always accompanied with some background noises. Speech processing and communication systems are to apply effective noise reduction techniques in order to extract the desired speech signal from its corrupted speech signal. That is, removal of background noise in the noisy speech. Some of noise reduction techniques are used in the speech processing like spectral subtraction, ceptral mean subtraction, blind equalization ,Adaptive wiener filtering ,Kalman filtering etc., are used various enhancement situations. Among this spectral subtraction is oldest one of the first algorithm proposed for removal of background noise. It is a single channel speech enhancement method for enhancement of speech degraded locale noise. The locale noise can disturb our conversation in a noisy environment like auditorium, street, market etc. This paper presents the performance of spectral subtraction algorithm is evaluate of a speech by signal to noise ratio value. Spectral subtraction algorithm is widely used in individual conversation due to its simplicity and implementation.
\end{abstract}

\section{General Terms}

Speech Enhancement, Filtering, VAD

\section{Keywords}

Spectral Subtraction, SNR, Noise Estimation

\section{INTRODUCTION}

Speech is a natural and basic way for humans to convey message and thoughts. Speech frequency normally ranges between $3 \mathrm{~Hz}$ to $4 \mathrm{KHz}$ depending upon the character. However the human beings have an audible frequency range of $20 \mathrm{~Hz}$ to $20 \mathrm{KHz}$. The most common problem in speech processing is the effect of meddling of noise in the speech signals. The noise masks the speech signal reduces the quality and the speech is greatly affected by presence of backdrop noise [3]. This make the listening task difficult for straight listeners and gives poor performance in some of speech processing like speech recognition, speech coder and speaker identification etc.,[16]. Noise shrinking or speech enrichment algorithm is to improve the performance of communication systems when their input or output signals are corrupted by noise signal.

The main objective of speech enhancement is to improve one or more perceptual aspects of speech such as class or clearness [14].The quality is a subjective measure that indicates the naturalness of the perceived speech and intelligibility is expected by the percentage of words that can be correctly identified by listeners [6]. The performance measures the excellence and intelligibility is very tough to satisfy at the same time. This paper presents speech enhancement method using spectral subtraction algorithm with their performance evaluation.

This paper is organized as follows, Section II describe speech enhancement method, Section III represent spectral subtraction method, Section IV gives the details implementation and methodology, Section V point up measures of performance, Section VI shows the experimental results ,Section VII deals the conclusion.

\section{SPEECH ENHANCEMENT METHODS}

There are various speech enhancement methods proposed for noise reduction and to improve the speech quality and clearness. Only one algorithm is not enough for all the types of noise present in the surrounding. Hence speech enhancement algorithms are created based on the applications. The speech enhancement systems can be classified in different types. That is single channel speech enhancement, multichannel speech enhancement and model based speech enhancement etc [8]. The single channel speech enhancement is very less pricey and make easy to process. Multi channel assists to eliminate noise in an effective manner and it is high difficult to process.

Speech enhancement is categorized in to three different ways (i) Filtering techniques (ii) Spectral restoration (iii) Model based methods [4]. i.e., spectral subtraction, signal space approach, Signal to KLT method etc., Among these filtering techniques spectral subtraction method is oldest one and easy to estimate the noise. Too many spectral subtraction methods are normally used for enhancement purpose like magnitude spectral subtraction, power spectral subtraction , spectral subtraction with over subtraction , Berouti spectral subtraction ,multiband spectral subtraction etc.[5], are used various situation of noisy speech removal.

The block diagram of speech enhancement is show in figure. 1 Speech noisy signal is segmented for $20-30 \mathrm{~ms}$ samples taken and windowed. 


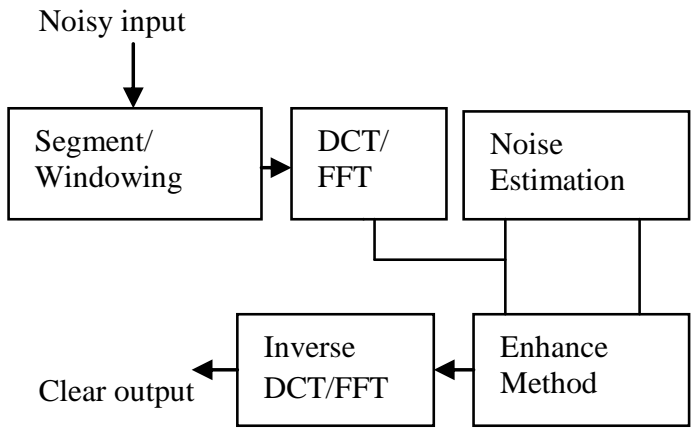

Fig.1.Block diagram of Speech Enhancement

Generally hamming window is used for speech is better. Then apply Fourier transform either Discrete or Fast Fourier transform of segmented and windowed. FFT is finest for speech enhancement. Noisy signal obtain and send to noise estimation block. This noise estimation block is used for calculate the overall noise in the original speech. Then if noise estimate is too low, unwanted residual noise will be audible else too high, speech will be unclear [11]. Enhancement block improve speech spectrum is generate and apply the inverse Fourier transform; it gives a clean speech signal.

\section{SPECTRAL SUBTRACTION METHOD}

Spectral subtraction is historically one of the oldest simple algorithm to implement easily and a minimal complexity of a speech enhacement. The spectral subtraction is based on the theory that the enhanced speech can be acquire by subtracting the estimated spectral factors from the continuum of the input noisy signal.

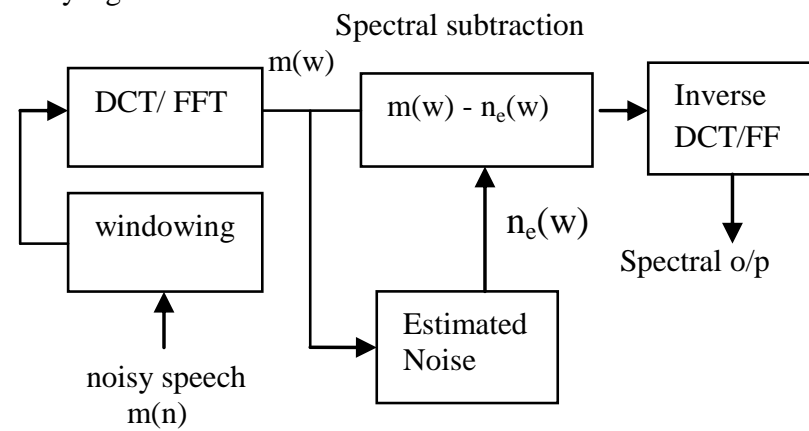

Fig.2.Block diagram of Spectral subtraction

Assuming $\mathrm{n}(\mathrm{s})$ is the noise signal, $\mathrm{c}(\mathrm{s})$ is clean speech signal and $\mathrm{u}(\mathrm{s})$ unclean noisy speech and it can be written as,

$$
\mathrm{u}(\mathrm{s})=c(s)+n(s) \text { for } 0 \leq s \leq S-1
$$

Where $s$ is the time index, $S$ is a number of samples. Generally speech enhancement is calculate enhanced speech $\mathrm{c}(\mathrm{s})$ from given $\mathrm{u}(\mathrm{s})$ with the guess that $\mathrm{n}(\mathrm{s})$ is uncorrelated with $\mathrm{c}(\mathrm{s})$.Input signal $\mathrm{u}(\mathrm{s})$ is segmented into $\mathrm{Z}$ segments of the same time-span. The subsequent equation frequency domain is changed from time domain as,

$$
\mathrm{U} z(w)=C z(w)+N z(w), \text { for } 0 \leq z \leq Z-1
$$

Where $\mathrm{Z}$ is the segment key and $\mathrm{U}_{\mathrm{z}}(\mathrm{w}), \mathrm{C}_{\mathrm{z}}(\mathrm{w}), \mathrm{N}_{\mathrm{z}}(\mathrm{w})$ are the Fourier magnitude taken from equation (1).If an estimate of noise continuum $\mathrm{NN}_{\mathrm{z}}$ can be obtained , then the speech $\mathrm{CC}_{\mathrm{z}}$ can be get from $\mathrm{U}_{\mathrm{z}}$

$$
C C z(w)=U z(w)-\propto N N z(w)
$$

In noise spectrum ,the none speech signal is estimated in the input signal. Voice activity detecter is used to find the quiet position in single channel speech enhancement. Here noise is imagine to be short ,so that noise from quiet frames can be used to remove noise from speech frames. The parameter $\alpha$ controls the amount of noise subtracted from the noisy signal. For full noise subtraction, $\alpha=1$ and for over-subtraction $\alpha>1$.

\section{IMPLEMENTATION}

Noisy speech signal which is degraded by noises at $10 \mathrm{~dB}$, $5 \mathrm{~dB}, 0 \mathrm{~dB}$ SNR is recorded in different environment. Sampling is carrying out at the rate of $16 \mathrm{~ms}$ and in each fragment it contains 128 samples. Hamming window is applied to the frame signal. The output frame chunk is given to DFT/FFT. After applying DFT/FFT, it calculates for the magnitude and phase for each frame. Then it verifies the entire clause and evaluates the noise estimation by using the method. Voice activity detector is utilize to ensure the voice or silence is present or not in the speech signal. After execute the entire operations next pace is to perform IDFT/IFFT to get back the new signal. Finally, realize the overlap and add method to the new signal to recover clear speech from noise speech.

$\begin{array}{cccc}\text { Noisy } & \text { Framing } & \text { Windowing } & \begin{array}{c}\text { Fourier } \\ \text { Transform }\end{array}\end{array}$

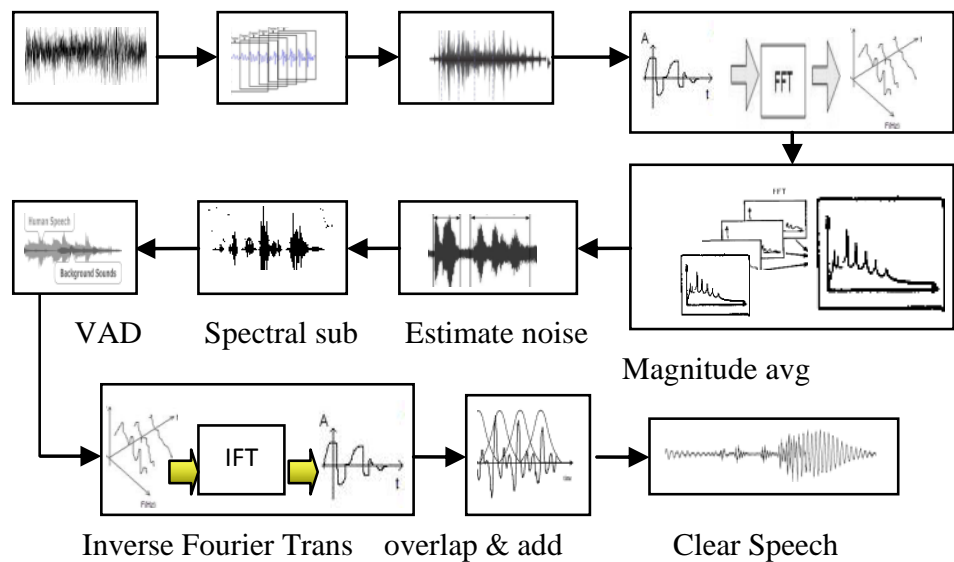

Fig.3.Flow diagram of Spectral Subtraction

\section{MEASURES OF PERFORMANCE}

\subsection{Signal-to-Noise Ratio (SNR)}

Signal-to-Noise Ratio (SNR)-to-noise ratio is a compute used in science and engineering that evaluates the level of a desired gesture to the level of background noise .Signal-to-noise ratio is sometimes used to refer to the part of useful information to extraneous data in a discussion or replace. 
Signal-to- noise ratio is defined as the power percentage between a significant background noises. SNR Values in $\mathrm{db}$.

$$
\mathrm{SNR}=10 \times \log _{10} \frac{\text { Mean }\left(\text { Input }^{2}\right)}{\text { Mean }\left(\text { Input }^{2}-\text { Enhanced }^{2}\right)}
$$

\section{EXPERIMENTAL RESULTS}

The process of the spectral subtraction algorithm was verify in Matlab7.0 and gives a recorded input of a street noise of $0 \mathrm{db}$, and $5 \mathrm{db}$. The spectral subtraction algorithm gives enhanced results. The figure shows the input noisy speech of $0 \mathrm{db}$ and the spectral subtraction output of $0 \mathrm{db}$.

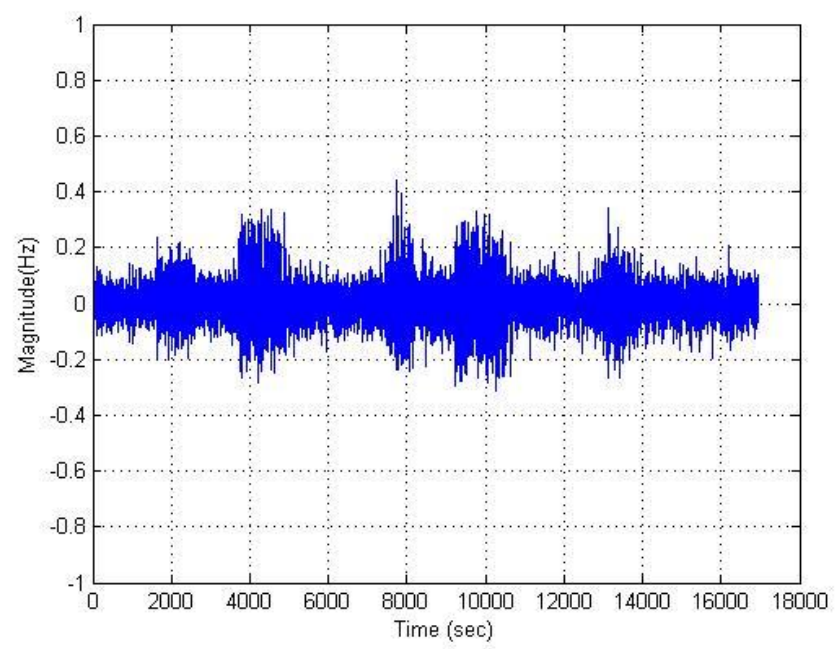

Fig.4. Original Speech of Odb

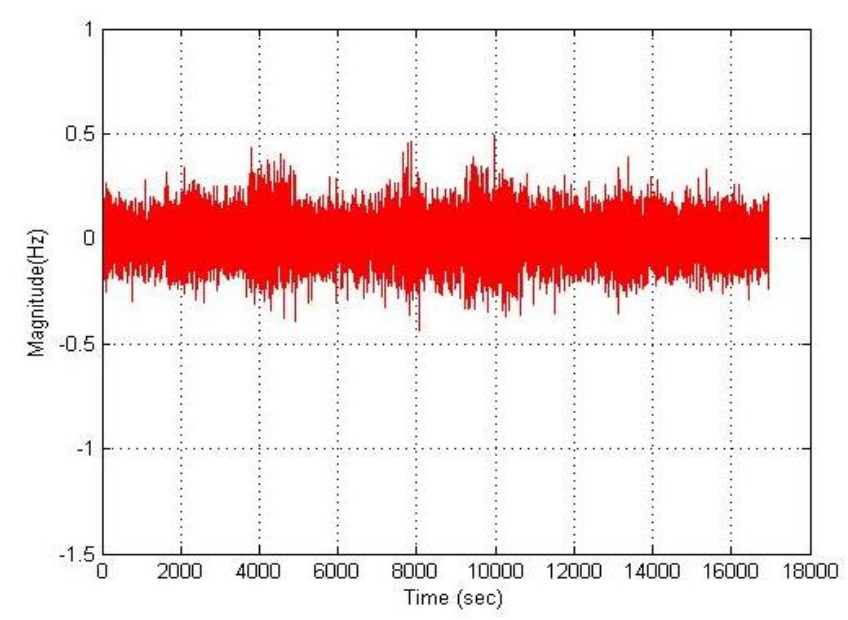

Fig.5.Noisy Speech of $0 \mathrm{db}$

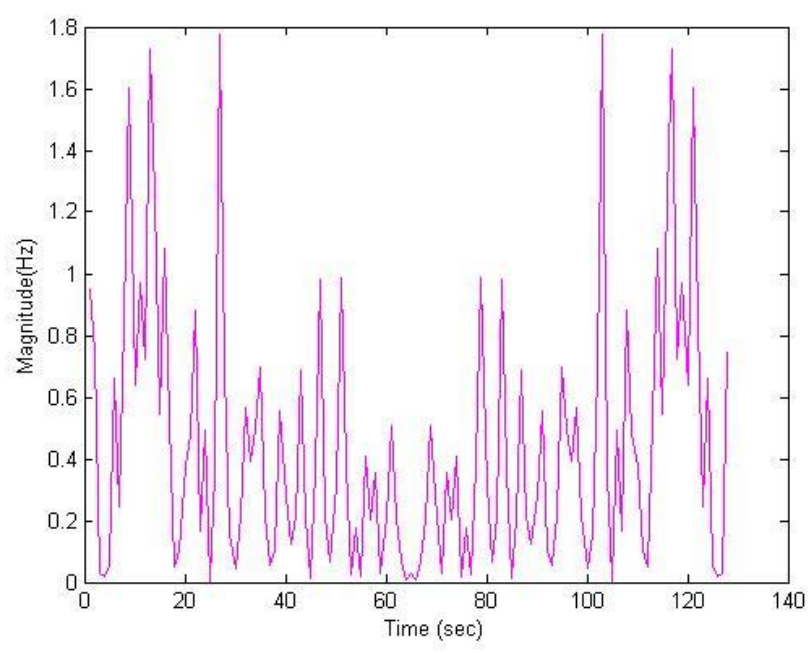

Fig.6. Magnitude Spectrum Speech of 0db

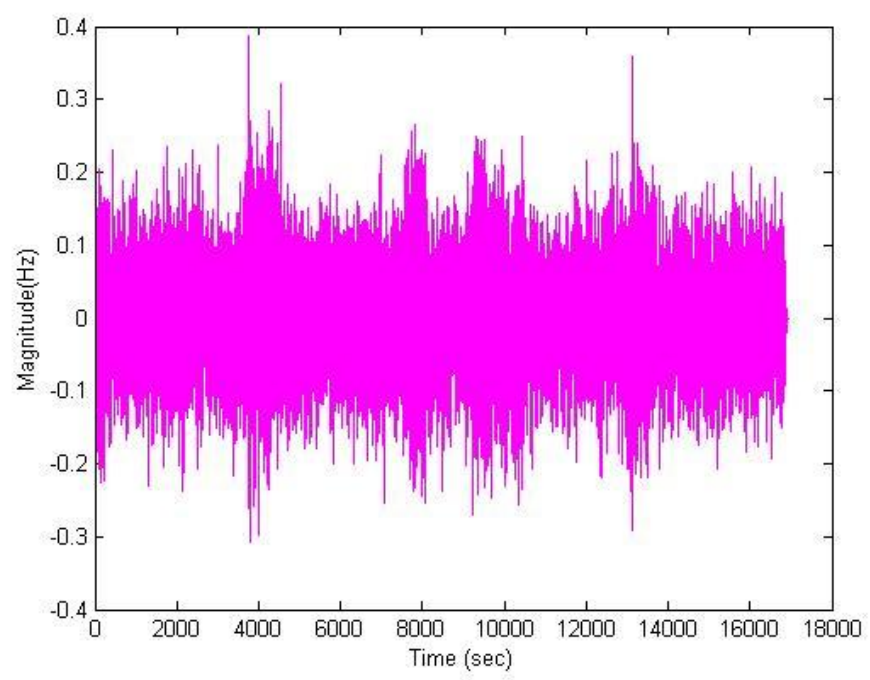

Fig.7. Spectral Filtered Speech output of 0db
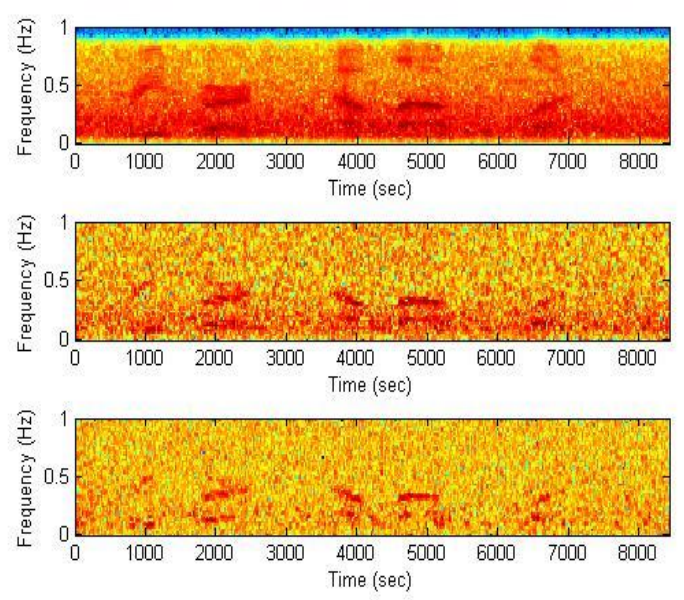

Fig.8. Spectrogram of filtered, noise, original of $0 \mathrm{db}$ 


\section{CONCLUSION}

This paper deals the spectral subtraction method of noise removing. And this document achieved the outcome of spectral subtraction method of noise removal to the desired goal. Even though there are so many problems to reduce the noise in spectral subtraction method with effective manner. In future, Suitable filter may be implementing to attain high quality of clear speech from the blare situation. So further study of noise removal and comparison is very much needs to achieve satisfaction. The concert of the spectral subtraction method is appraised by using signal to noise ratio analysis which gives satisfactory results.

\section{ACKNOWLEDGMENTS}

The author thanks the authorities of Annamalai University for providing the required facilities in order to complete this portion of work.

\section{REFERENCES}

[1] S.B.Magre, P.V.Janse, R.R.Deshmukh "A Review on Feature Extraction and Noise Reduction Technique" International Journal of Advanced Research in Computer Science and Software Engineering, ISSN: 2277 128X, Volume No.4, Issue No.2, pp: 352-356, 2014.

[2] Subha S, V.Murugan "Reverberation Suppression of Noisy Speech Signal" International Journal of Innovative Research in Computer and Communication Engineering, Vol.2, Special Issue 1, March 2014

[3] Rupali V. Mane, Dr.M.T.Kolte "Implementation of Adaptive Filtering Algorithm for Speech Signal on FPGA" International Journal of Innovative Research in Electrical, Electronics, Instrumentation and Control Engineering Vol. 2, Issue 3, March 2014

[4] Mrs.Vishakha V. Jadhav, Prof. Vijay M. Sardar "Improved Performance Based Method for Text Independent Speaker Identification" International Journal of Innovative Technology \& Adaptive Management (IJITAM) ISSN: 2347-3622, Volume-1, Issue-6, March, 2014

[5] Nainesh B Patel, Prof. Hardik N Patel "Single channel speech enhancement techniques for removal of additive noise" IOSR Journal of Electronics and Communication Engineeringe-ISSN: 2278-2834,p- ISSN: 22788735. Volume 9, Issue 2, Ver. III (Mar - Apr. 2014), PP $37-41$

[6] Ganga Prasad, Surendar "A Review of Different Approaches of Spectral Subtraction Algorithms for Speech Enhancement" Current Research in Engineering, Science and Technology (CREST) Journals, Vol 01। Issue 02 | April 2013 | 57-64

[7] Lalchhandami and Rajat Gupta "Different Approaches of Spectral Subtraction Method for Speech Enhancement" International Journal of Mathematical Sciences, Technology and Humanities 95 (2013) 1056 1062 ISSN 22495460
[8] Shishir Banchhor, Jimish Dodia, Darshana Gowda "GUI Based Performance analysis of speech enhancement techniques" International Journal of Scientific and Research Publications, Volume 3, Issue 9, September 20131 ISSN 2250-3153

[9] Suma. M. O, Madhusudhana Rao. D, Rashmi. H. N and Manjunath B.S "Speech Enhancement using Spectral Subtraction" International Journal of Advanced Electrical and Electronics Engineering, (IJAEEE) ISSN (Print), 2278-8948, Volume-2, Issue-4, 2013

[10]Isiaka A. Alimi, Michael O. Kolawole "Enhancement of Speech Communication Technology Performance Using Adaptive-Control Factor Based Spectral Subtraction Method" JTIT, 2/2013

[11]Anuprita P Pawar,.Kirtimalini. B.Choudhari "Enhancement of Speech in Noisy Conditions" International Journal of Advanced Research in Electrical, Electronics and Instrumentation Engineering Vol. 2, Issue 7, July 2013

[12]Ching-Ta Lu1, Kun-Fu Tseng and Chih-Tsung Chen "Reduction of Musical Residual Noise Using HybridMean Filter" International Journal of Signal Processing, Image Processing and Pattern Recognition Vol. 6, No. 4, August, 2013

[13]Arata Kawamura, Weerawut Thanhikam, and Youji Iiguni "Single Channel Speech Enhancement Techniques in Spectral Domain" International Scholarly Research Network ISRN Mechanical Engineering Volume 2012

[14]Ekaterina Verteletskaya, Boris Simak "Noise Reduction Based on Modified Spectral Subtraction Method" IAENG International Journal of Computer Science, , $38: 1$, IJCS

[15]Anuradha R. Fukane, Shashikant L. Sahare "Different Approaches of Spectral Subtraction method for Enhancing the Speech Signal in Noisy Environments" IJSER, Volume 2, Issue 5, May-2011

[16] Paurav Goel, Anil Garg "Review of Spectral Subtraction Techniques for Speech Enhancement" International Journal of Electronics \& Communication Technology Vol. 2, Issue 4, Oct. - Dec. 2011

[17] Radu Mihnea Udrea, Dragos Nicolae Vizireanu, Claudia Cristina Oprea, Ionut Pirnog "An Improved Multi-band Speech Enhancement Method for Colored Noise Estimation and Reduction" IJAT, vol 3 no 3 \& 4, year 2010

[18] Ekaterina Verteletskaya, Boris Simak "Enhanced spectral subtraction method for noise reduction with minimal speech distortion" IWSSIP 2010 - 17th International Conference on Systems, Signals and Image Processing

[19] D. Deepa, A. Shanmugam "Spectral Subtraction Method of Speech Enhancement using Adaptive Estimation of Noise with PDE method as a preprocessing technique" ICTACT Journal Of Communication Technology, March 2010, Issue: 01 